ARTIGO ORIGINAL

ISSN 1677-5090

(C) 2018 Revista de Ciências Médicas e Biológicas

DOI: http://dx.doi.org/10.9771/cmbio.v17i2.25590

\title{
Potencial antibacteriano e perfil farmacognóstico das folhas de Hibiscus acetosella Welw Ex Hiern
}

\author{
Antibacterial potential and pharmacognostic profile \\ of Hibiscus acetosella Welw Ex Hiern
}

\begin{abstract}
Paula da Silva Cardoso ${ }^{1 *}$, Fernanda Dagostim Mandalli², Tatiana Barichello ${ }^{3}$, Patricia de Aguiar Amaral ${ }^{4}$
${ }^{1}$ Mestre em Ciências Ambientais, Universidade do Extremo Sul Catarinense, Programa de Pós-Graduação em Ciências Ambientais - PPGCA; ${ }^{2}$ Graduada em Farmácia. Universidade do Extremo Sul Catarinense Departamento de Farmácia; ${ }^{3}$ Doutora em Ciências farmacêuticas. Universidade do Extremo Sul Catarinense, Programa de Pós-Graduação em

Ciências da Saúde - PPGCS; ${ }^{4}$ Doutora. Universidade do Extremo Sul Catarinense, Programa de Pós-Graduação em Ciências Ambientais - PPGCA
\end{abstract}

\begin{abstract}
Resumo
Introdução: as Hisbiscus pertencentes à família Malvaceae são amplamente utilizadas na área ornamental e vem ganhando espaço na área alimentícia com suas flores comestíveis e corantes naturais. Alguns estudos demonstraram atividade antibacteriana de algumas espécies deste gênero frente a diversos microorganismos. Hibiscus acetosella, também conhecida popularmente como vinagreira, possui em literatura científica pouca informação sobre sua composição química e ação antibacteriana. Objetivo: caracterizar o perfil farmacognóstico relacionando com a ação microbiológica das folhas de $\mathrm{H}$. acetosella. Metodologia: o perfil farmacognóstico foi realizado através de testes de precipitação, teste colorimétrico, quantificação de compostos fenólicos e análise cromatográfica do extrato hidroalcoólico e das frações das folhas de $\mathrm{H}$. acetocella. A ação antibacteriana do extrato hidroalcoólico $(60 \mathrm{mg})$ e frações $(25 \mathrm{mg})$ foi analisada frente aos microorganismos Staphylococcus aureus, Escherichia coli e Pseudomonas aeruginosa por meio do método de difusão em ágar. Resultados: a análise farmacognóstica apresentou resultados positivos para as classes de substâncias: taninos, flavonoides, cumarinas, heterosídeos cardiotônicos e alcaloides. O extrato hidroalcoólico possui aproximadamente $352,85 \mathrm{mg} / \mathrm{L}$ de polifenóis totais. As frações com caráter mais polar ( $n$-butanol e acetato de etila) apresentaram efeito relevante contra os microorganismos $S$. aureus e $P$. aeruginosa. Conclusão: os resultados demonstraram que ação antibacteriana pode estar relacionada com a classe de com compostos fenólicos, uma vez que as frações que apresentaram melhor resultado possuem maior concentração destes metabólitos.

Palavras-chave: Compostos Fenólicos. Staphylococcus Aureus. Pseudomonas Aeruginosa. Plantas Medicinais
\end{abstract}

\begin{abstract}
Introductio the Hisbiscus belonging to the family Malvaceae are widely used in the ornamental area and have been gaining space in the food area with its edible flowers and natural dyes. Some studies have demonstrated antibacterial activity of some species of this genus against different microorganisms. Hibiscus acetosella, also popularly known as vinegar, has little scientific information about chemical composition and antibacterial action. Objective: caracterizar o perfil farmacognóstico relacionando com a ação microbiológica das folhas de H. acetosella. Method: the pharmacognostic profile was performed through precipitation tests, colorimetric test, quantification of phenolic compounds and chromatographic analysis of the hydroalcoholic extract and the fractions of the leaves of $\mathrm{H}$. acetocella. The antibacterial action of the hydroalcoholic extract $(60 \mathrm{mg})$ and fractions $(25 \mathrm{mg})$ were analyzed against the microorganisms Staphylococcus aureus, Escherichia coli and Pseudomonas aeruginosa through the agar diffusion method. Results: the pharmacognostic analysis presented positive results for the classes of substances: tannins, flavonoids, coumarins, cardiotonic heterosides and alkaloids. The hydroalcoholic extract has approximately $352.85 \mathrm{mg} / \mathrm{L}$ of total polyphenols. The most polar fractions (n-butanol and ethyl acetate) had a significant effect against the S. aureus and P. aeruginosa microorganisms. Conclusions: the results showed that the antibacterial action may be related to the class of phenolic compounds, since the fractions presented the best antibacterial result have a higher concentration of these metabolites.
\end{abstract}

Keywords: Phenol Compounds. Staphylococcus Aureus. Pseudomonas Aeruginosa. Medicinal Plants.

\section{INTRODUÇÃO}

A falta de recursos e acesso às terapias modernas fazem com que diversas populações utilizem métodos tradicionais da medicina popular para realizar os cuidados

Correspondente/Corresponding: *Paula da Silva Cardoso - UNESC Universidade do Extremo Sul Catarinense - End: Av. Universitária, 1105 - Bairro Universitário, Criciúma, Santa Catarina - CEP: 88806-000. - Tel: (48)99623-6129 / (48)3431-2535 - E-mail: paulasc.far@gmail.com básicos à saúde. As preparações à base de plantas medicinais são uma das principais práticas utilizadas pelas pessoas em geral. Por conta disso, as plantas acabam exercendo um recurso vital para os cuidados primários a saúde (CALIXTO, 2000).

As atividades terapêuticas atribuídas às plantas medicinais estão relacionadas as moléculas do seu metabolismo secundário, que se diferencia em cada espécie para atender a uma função ecológica específica. Esta 
diversidade de moléculas faz das plantas uma fonte interessante para descoberta de moléculas bioativas e para o desenvolvimento de fármacos (JHEMES et al., 2017). Cientistas de todo o mundo vêm estudando e confirmando o potencial das plantas e de suas substâncias isoladas frente a diversos micro-organismos (MUNUSWAMY et al., 2013; SHARMA et al., 2017).

Ríos e Recio (2005) listaram espécies vegetais com ação antibacteriana, além de identificar que os compostos fenólicos foram os constituintes químicos predominantes nestas plantas, constatando que bactérias gram positivas são sensíveis a este grupo de substâncias.

Em um levantamento de espécies vegetais com ação antibacteriana promissora encontram-se as plantas Hibiscus esculentus, Hibiscus trionum e Hibiscus syriacus. Os extratos dessas espécies tem atividade antibacteriana em ensaios de diluição em caldo contra o microorganismo Mycobacterium tuberculosis (GAUTAM; SAKLANI; JACHAK, 2007). Outras espécies deste gênero como a Hibiscus sabdariffa e Hibiscus tiliacua têm ação antibacteriana frente aos microorganismos: Micrococcus luteus, Staphylococcus aureus, Pseudomonas aeruginosas, Salmonella choleroesuis e Escherichia coli (WONG; LIM; CHAN, 2010).

Dentre as espécies do gênero Hisbiscus encontra-se Hibiscus acetosella Welw Ex. Hiern. com origem africana popularmente conhecida como vinagreira, groselheira ou quiabo roxo. Tem como aspectos botânicos ser uma planta arbustiva, de caule semi-lenhoso, folhas cor de vinho escura com nervuras palmadas, flores solitárias de cor rosa arroxeada com frutos em cápsulas (LORENZI; SOUZA, 1999). As folhas de $H$. acetosella são também consumidas pela população na forma de salada e na produção de geleia. Há no entanto, poucas informações em literatura científica em relação à atividade antimicrobiana para esta espécie quando comparado com outras espécies de Hibiscus, como também em relação à sua composição fitoquímica. Portanto, este estudo objetiva investigar a ação antibacteriana e o perfil farmacognóstico de $\mathrm{H}$. acetosella.

\section{METODOLOGIA}

O material vegetal utilizado para as análises foram folhas de $\mathrm{H}$. acetosella coletadas no Horto Florestal da Universidade do Extremo Sul Catarinense (UNESC). A planta foi previamente identificada pela botânica Dra. Vanilde Citadini-Zanette do Herbário Pe. Dr. Raulino Reitz, da UNESC. Uma exsicata foi armazenada no mesmo Herbário, sob o registro CRI: 8551 . As folhas foram secas em estufa a $40^{\circ} \mathrm{C}$, trituradas e maceradas em álcool $70 \%$ na proporção de 1:5 (p/v), por 15 dias. Após este período o extrato foi filtrado e o solvente eliminado sob pressão reduzida, para a obtenção do extrato hidroalcoólico, nomeado de EH. Foi utilizada à metodologia de partição líquido-líquido no $\mathrm{EH}$ (8 g) com os solventes: diclorometano, acetato de etila e $n$-butanol. Após a extração, os solventes foram eliminados sob pressão reduzida. As frações foram respectivamente nomeadas de F-DCM, F-AcOEt e F-BuOH.
As amostras EH, F-DCM, F-AcOEt e F-BuOH foram analisadas para os seguintes metabólitos: compostos fenólicos, cumarinas, antraquinonas, taninos, flavonoides, saponinas, alcaloides e heterosídeos cardiotônicos.

A determinação dos níveis de polifenóis totais do EH, F-DCM, F-AcOEt e F-BuOH $(0,6 \mathrm{mg} / \mathrm{mL})$, foi realizada por meio do método Folin Ciocalteu. $\mathrm{O}$ ácido gálico foi utilizado na curva padrão nas concentrações de 250, 200, 150, 100 e $50 \mathrm{mg} / \mathrm{L}$. As leituras foram realizadas em espectrofotômetro a $765 \mathrm{~nm}$ em duplicata (SHAHIDI; NACZK, 1995). A equação de reta foi obtida a partir da regressão linear utilizando o programa Microsoft Excel ${ }^{\circledR}$.

Para realizar a identificação qualitativa das antocianinas, moléculas muito presentes em espécies de Hisbiscus (GRAJEDA-IGLESIAS et al., 2017) utilizou-se a técnica de cromatografia em coluna (CC) para isolamento e cromatográfica por camada delgada (CCD), seguida por revelação com anisaldeído sulfúrico. A CC foi realizada com EH em sílica gel 60F utilizando como fase móvel os solventes: hexano e AcOEt nas proporções 8:2, 1:1, 2:8, AcOEt e MeOH 6:4 e MeOH. As análises CCD foram realizadas com as frações F-DCM, F-AcOEt e F-BuOH e com as frações da CC.

Os eluentes utilizados para $\mathrm{CCD}$ foram: $\mathrm{CHCl}_{3}: \mathrm{AcOEt}$ (60:40), $\mathrm{CHCl}_{3}: \mathrm{MeOH}: \mathrm{CH}_{3} \mathrm{COOH}$ (47:47:5), AcOEt: $\mathrm{MeOH}$ (60:40), AcOEt: $\mathrm{MeOH}: \mathrm{H}_{2} \mathrm{O}$ (100: 13,5:10) e $n$-butanol: $\mathrm{CH}_{3} \mathrm{COOH}: \mathrm{H}_{2} \mathrm{O}$ (50:10:20). As CCD foram analisadas em lâmpada de UV no comprimento de onda $365 \mathrm{~nm}$ e algumas placas foram reveladas com anisaldeído sulfúrico (BLADT, 1996).

A atividade antimicrobiana foi realizada por meio do método difusão em ágar com disco de papel para o controle positivo ou em poço para o extrato hidroalcoólico, frações e controle negativo. $O$ teste foi realizado em duplicata. As bactérias Staphylococcus aureus (ATCC 6538), Pseudomonas aeruginosa (ATCC 27853) e Escherichia coli (ATCC 25922) foram inoculadas separadamente em ágar Müeller Hinton, Cetrimide ou MacConkey, respectivamente. A cultura bacteriana foi crescida a 37 으 por 24 horas. Após a incubação, a cultura foi diluída em solução salina estéril $(\mathrm{NaCl} 0,9 \%)$ de acordo com a escala de MacFarland $(0,5)$. Uma alíquota da suspensão foi inoculada em placa contendo ágar Müeller Hinton. Cada placa continha poços com 7 $\mathrm{mm}$ de diâmetro onde foi aplicado $60 \mu \mathrm{L}$ da amostra e cada placa recebeu um disco de ceftriaxona. As amostras testadas foram: EH $60 \mathrm{mg}$ ou F-DCM $25 \mathrm{mg}$ ou F-AcOEt $25 \mathrm{mg}$ ou F-BuOH $25 \mathrm{mg}$. A ceftriaxona foi utilizada como controle positivo e à solução de diluição das amostras (água com $3 \%$ de tween 80) como controle negativo. Após aplicação das amostras, as placas foram incubadas a 37 ㅇ por 24 horas. Depois do tempo de incubação verificou-se a presença do halo de inibição ao redor do material e mensurado seu diâmetro com auxílio de uma régua milimetrada (CLSI, 2009).

\section{RESULTADOS}

O EH obteve rendimento de $12,2 \%$, a partição líquido-líquido do $\mathrm{EH}$ resultou em maiores rendimentos quando 
realizados com os solventes, acetato de etila (F-AcOEt $20,1 \%$ ) e $n$-butanol (F-BuOH 12,5\%), comparados ao diclorometano (F-DCM 4,5\%).

Os resultados referentes à análise farmacognóstica do EH, F-DCM, F-AcOEt e F-BuOH estão descritos na Tabela 1. $\mathrm{O} E \mathrm{E}$ apresentou ausência de antraquinonas e saponinas,e presença dos compostos fenólicos, flavonoides, taninos, cumarinas, alcaloides e heterosídeos cardiotônicos. As frações F-AcOEt e F-BuOH obtiveram resultados positivos para os mesmos metabólitos do EH, já o F-DCM obteve resultados negativos para taninos e heterosídeos cardiotônicos.

Tabela 1 - Reações indicativas de substâncias presentes no extrato hidroalcoólico (HE), nas frações de butanol (F-BuOH), acetato de etila (F-ACOEt) e diclorometano (F-DCM).

\begin{tabular}{|c|c|c|c|c|c|}
\hline & HE & F-BuOH & F-AcOEt & F-DCM & Reações \\
\hline Substâncias Fenólicas & + & + & + & + & Cloreto férrico; Hidróxido de potássio \\
\hline Flavonoides & + & + & + & + & Óxido de magnésio; Ácido clorídrico \\
\hline Taninos & + & + & + & - & Cloreto férrico; Solução de gelatina \\
\hline Cumarinas & + & + & + & + & Reativo de $\mathrm{KOH}$; Visualização UV \\
\hline Antraquinonas & - & & & & Hidróxido de potássio \\
\hline Saponinas & - & & & & Água destila e agitação em cilindro graduado; Ácido clorídrico. \\
\hline \multirow[t]{4}{*}{ Alcaloide } & + & - & - & - & Reativo de Mayer \\
\hline & + & + & + & + & Reativo de Dragendorf \\
\hline & + & + & - & + & Reativo de Brouchardat; \\
\hline & + & - & - & - & Reativo de Bertrand \\
\hline \multirow[t]{2}{*}{ Heterosídeos cardiotônicos } & + & + & + & - & Reação de Baljet; \\
\hline & + & + & + & - & Reação de Keller-Kiliani; \\
\hline
\end{tabular}

(+) resultado positivo para reação; (-) resultado negativo para reação; (EH) extrato hidroalcoólico da folhas de H. acetosella; (F-DCM) fração de diclorometano; (F-AcOEt) fração de acetato de etila; (F-BuOH) fração de $n$-butanol; as três frações foram obtidas através do fracionamento líquidolíquido do $\mathrm{EH}$.

Fonte: Autores

O perfil cromatográfico das três frações da partição líquido-líquido (F-DCM, F-AcOEt, F-BuOH) se diferenciou tanto na coloração como no fator de retenção $\left(R_{f}\right)$ das manchas. De todos os eluentes testados $n$-BuOH: $\mathrm{CH}_{3} \mathrm{COOH}: \mathrm{H}_{2} \mathrm{O}$ (50:10:20) foi o mais eficiente para obtenção do melhor perfil cromatográfico em CCD. As placas reveladas com anisaldeído sulfúrico apresentaram na F-BuOH várias manchas de coloração avermelhada. Conforme Bladt (1996) o surgimento desta coloração caracteriza a presença de antocianinas, a F-AcOEt também apresentou uma macha avermelhada.

A cromatografia em coluna do EH produziu 26 frações avaliadas por CCD, por isso as com perfil cromatográfico semelhantes foram reagrupadas dando um total de 6 frações nomeadas de A, B, C, D, E e F. A fração E apresentou apenas uma mancha, que quando revelada com anisaldeído apresentou coloração roxa caracterizando a presença de antocianina, conforme Bladt (1996).

No doseamento de compostos fenólicos a curva padrão com ácido gálico gerou a equação da reta $\mathrm{y}=$ $0,001 x+0,014$, com coeficiente de correlação de 0,997. Os testes foram realizados em duplicata e os resultados estão descritos em mg/L (Tabela 2).

$\mathrm{O}$ teste microbiológico realizado com as frações F-BuOH e F-AcOEt demonstra ação antibacteriana relevante frente aos microorganismos $S$. aureus e $P$. aeruginosa; já a F-DCM foi ineficaz contra as bactérias testadas. $\mathrm{OEH}$ diminuiu o crescimento das três espécies de bactérias testadas. Os diâmetros em milímetros $(\mathrm{mm})$ dos halos de inibição estão descritos na Tabela 3.

Tabela 2 - Concentração de polifenóis totais em $\mathrm{mg} / \mathrm{L}$.

\begin{tabular}{lc}
\hline Amostras $(0,6 \mathrm{mg} / \mathrm{mL})$ & Concentração polifenóis totais - $\mathrm{mg} / \mathrm{L}$ \\
\hline F-AcOEt & 352,85 \\
\hline F-BuOH & 224,77 \\
\hline F-DCM & 254,38 \\
\hline EH & 249,77
\end{tabular}

(EH) extrato hidroalcoólico da folhas de H. acetosella; (F-DCM) fração de diclorometano; (F-AcOEt) fração de acetato de etila; (F-BuOH) fração de $n$-butanol; as três frações foram obtidas através do fracionamento líquido-líquido do $\mathrm{EH}$.

Fonte: Autores.

Tabela 3 - Diâmetros dos halos de inibição em $\mathrm{mm}$ das frações provindas do fracionamento líquido-líquido $\mathrm{da} \mathrm{H}$. acetosella.

\begin{tabular}{lcccc}
\hline Amostras & Concentração & E. coli & S. aureus & P. aeruginosa \\
\hline Padrão (+) & $30 \mathrm{mg}$ & $30 \mathrm{~mm}$ & $24 \mathrm{~mm}$ & $17 \mathrm{~mm}$ \\
\hline Padrão (-) & $60 \mu \mathrm{L}$ & $0 \mathrm{~mm}$ & $0 \mathrm{~mm}$ & $0 \mathrm{~mm}$ \\
\hline F-AcOEt & $25 \mathrm{mg}$ & $5 \mathrm{~mm}$ & $24 \mathrm{~mm}$ & $11 \mathrm{~mm}$ \\
\hline F-BuOH & $25 \mathrm{mg}$ & $0 \mathrm{~mm}$ & $19 \mathrm{~mm}$ & $12 \mathrm{~mm}$ \\
\hline F-DCM & $25 \mathrm{mg}$ & $0 \mathrm{~mm}$ & $0 \mathrm{~mm}$ & $0 \mathrm{~mm}$ \\
\hline EH & $60 \mathrm{mg}$ & $16 \mathrm{~mm}$ & $21 \mathrm{~mm}$ & $19 \mathrm{~mm}$ \\
\hline
\end{tabular}

Padrão (+): Ceftriaxona; Padrão (-): Água e tween 80 a 3\%; (EH) extrato hidroalcoólico da folhas de H. acetosella; (F-DCM) fração de diclorometano; (F-AcOEt) fração de acetato de etila; (F-BuOH) fração de $n$-butanol; as três frações foram obtidas através do fracionamento líquido-líquido do $\mathrm{EH}$. Fonte: Autores. 


\section{DISCUSSÃO}

As análises de precipitação e colorimétrica das frações mostraram que a F-DCM se diferencia das demais frações (F-AcOEt e F-BuOH) quanto à composição fitoquímica. Obtiveram-se resultados positivos no EH, F-AcOEt e F-BuOH para os seguintes compostos: fenólicos, taninos, flavonoides, cumarinas, heterosídeos cardiotônicos e alcaloides. A F-DCM obteve resultados positivos para compostos fenólicos, flavonoides, cumarinas e alcaloides. Estes resultados corroboram com outros estudos que já identificaram heterosídeos cardiotônicos em outras espécies do gênero Hisbiscus (MUNGOLE; CHATURVEDI, 2011). Estes compostos já foram identificados em outros gêneros como, por exemplo, Corchorus da família Malvaceae, que foi isolado e identificado o composto estrofantina (AL-SNAFI, 2016). A presença de alcaloides já foi detectada também na Hibiscus vitifolius (RAMASAMY; SARASWATHY, 2014), (RAMASAMY; SARASWATHY, 2014) contribuindo com os resultados positivos obtidos para esta classe de metabólito presente na H. acetocella.

As placas reveladas com anisaldeído sulfúrico apresentaram na $\mathrm{F}-\mathrm{BuOH}$ várias manchas de coloração avermelhada. De acordo com Bladt (1996), o surgimento desta coloração caracteriza a presença de antocianinas, ocorrendo o mesmo resultado na F-AcOEt.

A F-DCM apresentou duas manchas de coloração alaranjada (UV), o que é característico de flavonóides. Quando utilizado o solvente clorofórmio: metanol: ácido acético glacial para identificação de alcalóides, além das manchas alaranjadas, também sugiram manchas de coloração amarela, rosa e azul (UV), sendo que a cor azul, conforme Bladt (1996) é indicativo de alcaloides. A F-AcOEt apresentou manchas de coloração amarela, alaranjada, verde e azul clara (UV). Para Bladt (1996), característico de flavonoides e alcaloides. A F-BuOH apresentou manchas de coloração azul clara e verde escura (UV), confirmando a presença de flavonoide e alcaloides.

A fração $E$ da cromatografia em coluna apresentou apenas uma mancha, que quando revelada com anisaldeído apresentou coloração roxa caracterizando a presença de antocianina, conforme Bladt (1996).

O EH apresentou aproximadamente $352,85 \mathrm{mg} / \mathrm{L}$ de polifenóis, que corresponde a aproximadamente $7 \%$ da composição das folhas seca. Os compostos fenólicos são produzidos pelo metabolismo secundário com a finalidade de defesa a insetos, patógeno e demais inimigos ambientais. Os níveis elevados destas substâncias na planta influenciam no aumentam a resistência a patógenos e insetos (LATTANZIO et al., 2006).

As F-BuOH e F-AcOEt testadas na concentração de 25 $\mathrm{mg}$ têm ação antibacteriana relativamente semelhantes aos resultados do $\mathrm{EH}$ frente ás bactérias $S$. aureus e $P$. aeruginosas, que tiverem halo de inibição de $21 \mathrm{~mm}$ e $19 \mathrm{~mm}$, respectivamente. Em relação à $E$. coli o EH gerou $16 \mathrm{~mm}$ de halo de inibição; já as frações não foram eficientes, pois apenas a F-AcOEt gerou halo de inibição de apenas $5 \mathrm{~mm}$ de diâmetro. O perfil fitoquímico da F-DCM apresentou resultados negativos para taninos e heterosídeos cardiotônicos, como também foi a única fração da extração líquido-líquido que não apresentou nas análises de CCD machas sugestivas de antocianinas, portanto a ausência destes componentes pode justificar sua ineficácia.

Dentre estes metabólitos secundários os polifenóis se destacam em relação à atividade antibacteriana, pois este grupo de moléculas é sintetizado pelas plantas para proteção contra patógenos, sendo as moléculas mais relevantes os flavonoides e os taninos (HAVSTEEN, 2002).

Estudos demonstram o potencial antibacteriano dos extratos com alto teor de polifenóis ou isolados, porém seu mecanismo de ação ainda não está bem esclarecido. Alguns autores têm sugerido que sua ação está relacionada com inibição de proteínas, enzimas e interação com elementos da membrana alterando sua permeabilidade e fluidez (CUSHNIE; LAMB, 2005). Os flavonoides possuem grande atividade sobre as bactérias gram positivas, pois são capazes de penetrar a membrana fosfolipídica e exercer função dentro da bactéria (DAGLIA, 2012).

A análise quantitativa de polifenóis de plantas utilizadas popularmente demonstrou que as plantas com maior concentração de taninos eram aquelas utilizadas com antibióticos (SIQUEIRA et al., 2012). Outros metabólitos secundários como os alcaloides (MOREL et al., 2005) também possuem estudos que relatam sua ação frente a bactérias gram positivas e gram negativas, sem deixar de descartar a possibilidade do sinergismo entre as moléculas.

\section{CONCLUSÃO}

H. acetosella é uma fonte interessante de polifenóis, tendo aproximadamente $7 \%$ de polifenóis totais nas folhas secas. Ainda se constatou a presença dos seguintes metabólitos: taninos, flavonoides, cumarinas, heterosídeos cardiotônicos e alcaloides.

As frações F-BuOH e F-AcOEt têm maior atividade antibacteriana frente às bactérias $S$. aureus e $P$. aeruginosa. A fração F-AcOEt na concentração de $25 \mathrm{mg}$ demonstrou ação idêntica à ceftriaxona para o microorganismo $S$. aureus com halo de inibição de $24 \mathrm{~mm}$ para ambas. Os resultados demonstraram que a ação antibacteriana pode estar relacionada à composição química dos extratos, já que a F-DCM foi a que mais se diferenciou das demais tanto no rendimento como na ausência de taninos, alcaloides e antocianina.

\section{AGRADECIMENTOS}

À professora Dra. Vanilde Citadini Zanette pela identificação botânica e ao Horto florestal da Universidade do Extremo Sul Catarinense por fornecer $H$. acetosella.

\section{REFERÊNCIA}

AL-SNAFI, A. E. The contents and pharmacological importance of corchorus capsularis- a review. J. Pharm., [s.l], v. 6, n. 6, p. 58-63, 2016. 
BLADT, W. H. Plant drug analysis - a thin-layer chromatography atlas. 2 ed. Berlin: Springer, 1996.

CLINICAL \& LABORATORY STANDARDS INSTITUTE (CLSI). Performance Standards for Antimicrobial Disk Susceptibility Tests. 10 Ed. Wayne, PA: Clinical and Laboratory Standards Institute, 2009.

CUSHNIE, T. P. T.; LAMB, A. J. Antimicrobial activity of flavonoids. Int. J. Antimicrob. Agents, Amsterdam, v. 26, n. 5, p. 343-356, 2005.

DAGLIA, M. Polyphenols as antimicrobial agents. Curr. Opin. Biotechno., v. 23, n. 2, p. 174-181, 2012.

GAUTAM, R.; SAKLANI, A.; JACHAK, S. M. Indian medicinal plants as a source of antimycobacterial agents. J. Ethnopharmacol., Limerick, v. 110, n. 2, p. 200-234, 2007.

GRAJEDA-IGLESIAS, C. et al. Lipophilization and MS characterization of the main anthocyanins purified from hibiscus flowers. Food Chem., Barking, v. 230, p. 189-194, 2017.

HAVSTEEN, B. H. The biochemistry and medical significance of the flavonoids, Pharmacol Ther., Oxford, v. 96, n. 2-6, p.67-202, 2002.

JHEMES, I. et al. a Diversidade da flora Brasileira No Desenvolvimento De Recursos De Saúde the Diversity of Brazilian Flora in the Development of Health resources. Revista Uningá, v. 31, n. 1, p. 35-39, 2017.

LATTANZIO, V. et al. Role of phenolics in the resistance mechanisms of plants against fungal pathogens and insects. Phytochemistry, New York, v. 661, n. 2, p. 23-67, 2006.

LORENZI, H.; SOUZA, H. M. Plantas ornamentais no Brasil: arbustivas, herbáceas e trepadeiras. 2.ed. Nova Odessa, SP: Plantarum, 1999. 1088 p.
MOREL, A. F. et. al. Cyclopeptide alkaloids from Scutia buxifolia Reiss and their antimicrobial activity. Phytochemistry, New York, v. 66, p. 2571-2576, 2005.

MUNGOLE, A.; CHATURVEDI, A. Hibiscus sabdariffa la rich source of secondary metabolites. Int. J. Pharm. Sci. Rev. Res., Bangarole, v. 6, n. 1, p. 83-87, 2011.

MUNUSWAMY, $H$. et al. A review on antimicrobial efficacy of some traditional medicinal plants in Tamilnadu. J Acute Disease, [s.l], v. 2, n. 2, p. 99-105, 2013.

RAMASAMY, D.; SARASWATHY, A. Vitiquinolone - A quinolone alkaloid from Hibiscus vitifolius Linn. Food Chem., Barking, v. 145, p. 970-975, 2014.

RÍOS, J. L.; RECIO, M. C. Medicinal plants and antimicrobial activity. J. Ethnopharmacol., Limerick, v. 100, n. 1-2, p. 80-84, 2005.

SHAHIDI, F.; NACZK, M. Food phenolics: sources, chemistry, effects, applications, technomic. Lanchester: Company Inc, 1995. p. 235-273.

SHARMA, A. et al. Antibacterial activities of medicinal plants used in Mexican traditional medicine. J. Ethnopharmacol., Limerick, v. 208, p. 264-329, 2017.

SIQUEIRA, C. F. D. Q. et al. Levels of tannins and flavonoids in medicinal plants: Evaluating bioprospecting strategies. J. Evid. Based Complementary Altern. Med., [s.I], v. 2012, p. 1-7, 2012.

WONG, S. K.; LIM, Y. Y.; CHAN, E. W. C. Evaluation of antioxidant, antityrosinase and antibacterrial activities of selected Hibiscus species. Ethobot. Leaflets, [s.I], v. 14, p.781-796, 2010.

Submetido em: $16 / 02 / 2018$

Aceito em: 29/08/2018 